\title{
CORRESPONDENCE
}

\section{Biosimilars or semi-similars?}

\section{To the Editor:}

It has now been more than a year since the approval of the first biosimilar monoclonal antibody $(\mathrm{mAb})$ was noted in your pages ${ }^{1}$. And yet the European Medicines Agency (EMA) still has not clarified the scientific basis for when in vitro data will be sufficient to claim extrapolation for a biosimilar approved in one indication for another.

Extrapolation is the pillar of the biosimilar regulatory pathway. Without extrapolation of biomarkers to clinical endpoints, of safety data to patient populations and, especially, of one indication to another, no abridged procedure is possible. And without

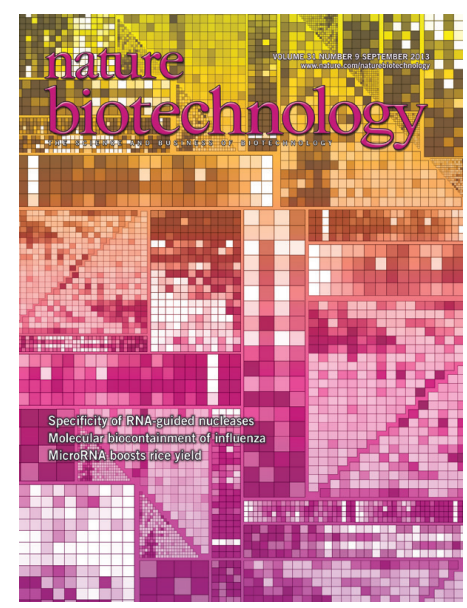

such scientific discussions because these products are all homologs of natural growth-regulating factors, used mainly as supplemental therapy. And, therefore, the original reference products are themselves human-made copies of endogenous

proteins and can be considered as the original biosimilars.

However, the situation is different for the next generation of biosimilars-mAb biosimilars. Therapeutic mAbs are used to neutralize regulating factors such as tumor necrosis factor- $\alpha$ (TNF- $\alpha$ ) and modulate receptor activity and for other biological activities not normally exerted by antibodies. an abridged procedure, there is no major cost reduction in obtaining marketing authorization.

The biosimilar guidelines of regulatory agencies worldwide request scientific justification for extrapolation. The EMA is the regulatory authority with most experience in biosimilars and has admitted initially four product classes (filgrastims, epoetins, human growth hormones and follicle-stimulating hormones) allowing extensive extrapolation. In two cases, the extrapolation of immunogenicity data obtained in low-risk cancer patients and/or by intravenous treatment was denied to high-risk patients treated subcutaneously and without concomitant immunosuppressive treatment ${ }^{2,3}$.

The justification of the extrapolation with this first generation of biosimilars allowed by the EMA is discussed in European Public Assessment Reports (EPARs; http://www.ema. europa.eu/ema/index.jsp? curl=pages/ medicines/landing/epar_search. jsp\&mid=WC0b01ac058001d125). Extrapolation is not a major issue in
In contrast with the first generation of biosimilars, mAbs are mostly disease modifying and active locally in the diseased organs. Also, some of the most commercially successful mAbs (such as infliximab and adalimumab) have multiple indications in a variety of diseases of which the pathogenesis is only partly understood and the mode of action largely unknown.

In 2013, the first biosimilar $\mathrm{mAb}$, infliximab, received marketing authorization in Europe for all indications of the reference product. The EPAR for this product was published with the justification of the extrapolation of the clinical data obtained in rheumatoid arthritis and ankylosing spondylitis to inflammatory bowel disease and psoriasis ${ }^{4}$. Surprisingly, comparative in vitro data were considered sufficient to claim extrapolation, but the significance of these data for the different indications is unknown. Accepting in vitro data as surrogates for clinical activity is also in contradiction with another pillar of the biosimilar approach: the need for clinical data to show therapeutic equivalence.
The companies involved in the above decision committed to a post-marketing comparative clinical trial comparing reference and biosimilar products in Crohn's disease. This extraordinary position of the EMA poses intriguing regulatory questions. What to do if the trials fail to show clinical equivalence for the biosimilar in Crohn's disease? Scientific logic says that the product should lose all indications, as biosimilarity is not shown. Other issues are the legal status of the indication and the nomenclature. Who is responsible if the current treatment of patients with Crohn's disease with the infliximab biosimilar proves unsafe and/ or less effective in the post-marketing trial? And according to the EMA's position, the term biosimilar should be restricted to products authorized on the basis of a comprehensive similarity exercise including comparative clinical data. Maybe 'semi-similar' is a better term for a product for which an indication is pending and a major part of the clinical comparative data is missing.

The EMA has pioneered the regulatory approach for biosimilars and has been a visionary example for regulators worldwide. Its set of guidelines has resulted in the introduction of safe and effective products, but mainly because they were copies of physiological protein factors. It is doubtful whether this also gives a scientific basis for the introduction of biosimilar $\mathrm{mAbs}$. Clarifying this issue is important; it is not just Europe and developed countries that need affordable, highquality biosimilar products, but developing countries as well. A new scientific paradigm is needed to make this possible. Considering the poor quality of the revised biosimilar guidelines ${ }^{3}$, the EMA should move to provide further clarification on this issue.

\section{COMPETING FINANCIAL INTERESTS}

The authors declare competing financial interests: details are available in the online version of the paper (doi:10.1038/nbt.3083). 


\section{Huub Schellekens \& Ellen Moors}

Department of Innovation, Utrecht University, Utrecht, the Netherlands, and Department of Pharmaceutical Sciences, Utrecht University, Utrecht, the Netherlands.

e-mail:h.schellekens@uu.nl

1. 1. Dorey, E. Nat. Biotechnol. 31, 774 (2013).

2. European Medicines Agency. CHMP Assessment Report for Filgrastim Hexal. (no. EMEA/CHMP/651324/2008) http://www.ema.europa.eu/docs/en_GB/document_library/EPAR_-_Public_assessment_report/ human/000918/WC500022471.pdf (2008).

3. European Medicines Agency. European Public
Assessment Report for Binocrit. http://www. ema.europa.eu/docs/en_GB/document_library/ EPAR_-_Scientific_Discussion/human/000725/ WC500053615.pdf (2007).

4. European Medicines Agency. CHMP Assessment Report for Remsima. (no. EMA/CHMP/589317/2013) http:// www.ema.europa.eu/docs/en GB/document library/ EPAR_-_Public_assessment_report/human/002576/ WC500151486.pdf (2013).

5. European Medicines Agency. CHMP Draft Guideline on Similar Biological Medicinal Products Containing Biotechnology-Derived Proteins as Active Substance: Non-Clinical and Clinical Issues. (no. CHMP EMEA/ CHMP/BMWP/42832/2005, rev. 1) http://www.ema. europa.eu/docs/en_GB/document_library/Scientific_ guideline/2009/09/WC500003920.pdf (2006).

\section{Outcubation-where incubation meets outsourcing}

\section{To the Editor:}

As highlighted in your February 2014 issue ${ }^{1}$, the pharma industry is increasingly relying on externalization efforts to supplement its inhouse R\&D activities ${ }^{2}$. Such activities have a long history and had been taking place within pharmaceutical companies long before Henry Chesbrough formulated his open innovation paradigm in 2003 (ref. 3). Indeed, there is an ample literature discussing the models and impact of open innovation approaches for the pharma industry ${ }^{4,5}$. In the following Correspondence, we describe the design and implementation of a new model of open innovation between academia and industry-a model we term 'outcubation'. This model is designed to overcome the disadvantages of existing innovation models, provides an alternative path for young scientists

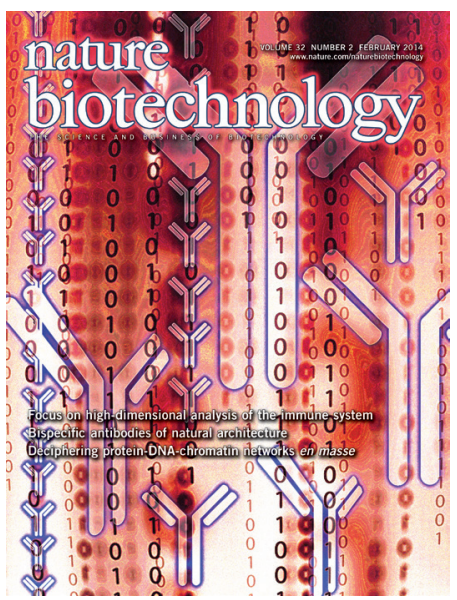

of scouting, due diligence, internalization of the asset and licensing fees can be very high compared with expenses in other innovation approaches. The major challenges of the M\&A approach are integration issues and decreased $R \& D$ productivity of the merged organizations ${ }^{6}$. The CVC approach usually does not exploit the potential synergy between internal and external resources. With crowdsourcing approaches, which are becoming increasingly popular, the quality of the submitted solutions is highly variable, and substantial screening and evaluation efforts are required.

To overcome the disadvantages of the models described above, we have created a new type of academicindustry partnership that synergizes the strengths of both. In this model, a company formulates a challenge (or topic) open to opportunities outside of academic tenure and combines synergistically the best of the academic and industry worlds.

Today, typical models for exploiting external innovation resources include outsourcing to contract research organizations (CROs), research collaborations with academic institutions, crowdsourcing, corporate venture capital (CVC), in-licensing and mergers and acquisitions (M\&A). Each approach has drawbacks; for example, outsourcing to a CRO may lead to a lack of innovative input, whereas an academic collaboration may not have enough oversight by the industry partner to be productive. With in-licensing, the cost a five-day boot camp. Candidates are divided into diverse teams and, with guidance from experienced mentors and with relevant business intelligence (e.g., access to the science and intellectual property literature and to market research data), develop competitive project proposals. On day five, they present their project proposals to a jury comprising senior management of the pharma company. The most attractive project proposal and the most talented candidates are selected, and the candidates receive a two- to four-year fellowship to work on their project.

The selected academic talents are then employed by and relocated to an outcubator, which is incorporated as a private biotech company. The outcubator is located on the campus of a global innovation hub, such as Heidelberg, Germany, and includes access to a state-of-the-art cell and molecular biology laboratory, office space and social space. Each selected team is sponsored by a pharma partner and guided by an experienced industry mentor (e.g., senior researcher of the sponsor company) and an academic mentor (e.g., a local professor from the specific field of the team's research topic). The academic mentor is proposed by the pharma partner and also is involved in team member selection. A team usually consists of a group leader (an experienced postdoctoral researcher with outstanding accomplishments in the field), two or three postdocs and two or three postgraduates or technicians.

With their location at innovation hotspots, outcubators are well integrated in the scientific environment, and resident teams benefit from stimulating discussions, exchange and collaborations within a highperforming ecosystem. The fact that guidance for the teams is provided by experienced mentors from both industry and academia ensures optimal support, combining academic curiosity, creativity and scientific excellence with product-oriented and efficiency-driven thinking and stringent quality-control systems.

Team members are driven by the research topic and the desire to conduct productoriented research. The goal is to combine the quest for fundamental understanding with practical usability in the frame of a new product or application. This is termed "working in Pasteur's quadrant" 7 and has been defined as a key target area by other successful innovation agencies, such as the US Defense Advanced Research Projects Agency (DARPA; Washington, DC) $)^{8}$. The innovation team should be intrinsically motivated, but it should also be supported by successful participation modules, as well as career- 